Citation: Kurulay, M., Senturk, I., "Numerical Solution of Time and Space Fractional Burger's Equation with Finite Difference Method". Journal of Engineering Technology and Applied Sciences 4 (2) 2019 : 71-83.

\title{
NUMERICAL SOLUTION OF TIME AND SPACE FRACTIONAL BURGER'S EQUATION WITH FINITE DIFFERENCE METHOD
}

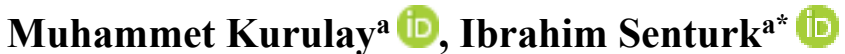 \\ ${ }^{a}$ Department of Mathematical Engineering, Yildiz Technical University, Istanbul, Turkey \\ mkurulay@yildiz.edu.tr,ibrahimsenturkmat@gmail.com (*corresponding author)
}

\begin{abstract}
In this study, fractional Burger's Equation, which has Dirichlet Boundary Conditions, is solved with the Finite Difference Method. Fractional Burger Equation is found by S. Momani, which is made with changing time and space terms with fractional terms. This equation is solved with the finite difference method and analysis of this scheme is discussed with examples. Stability and Uniqueness are discussed with using matrix method. We compare analytical and numerical solutions with error analysis of them.
\end{abstract}

Keywords: Finite difference method, Burger's equation, fractional derivative

\section{Introduction}

Burgers' equation [1] is a famous non-linear equation for physics problems. The problem has Dirichlet boundary conditions. With changing the order of differential terms of the equation with fractional order, we can achieve the fractional Burger's Equation [12] which was formulated by S. Momani. The following equation is fractional Burger's Equation:

$$
\begin{aligned}
& \frac{\partial^{\alpha} u}{\partial t^{\alpha}}+u \frac{\partial u}{\partial x}=v \frac{\partial^{\beta} u}{\partial x^{\beta}} \quad a \leq x \leq b, 0<t \leq T, \\
& 0<\alpha<1,1<\beta<2
\end{aligned}
$$

The problem has the following conditions: 
boundary conditions :

$u(a, t)=f_{1}(t), \quad u(b, t)=f_{2}(t)$

initial condition :

$u(x, 0)=f(x)$

where $v>0$ is the viscosity constant, $f_{1}(x), f_{2}(x)$ and $f(x)$ are the functions of $x$. There are many studies about the solving of Burger's and Fractional Burger's equations. Some finite difference approximations are found in the literature[16-17]. For example Zhang and Wang, Kutluay and Bahadir and Özdes, Pandey and Verma studied on finite difference method for burger's equation [3, 5 and 6], Varöglu and LiamFinn studied with finite elements method for solve Burger's equation [4]. Momani and Kurulay have studies about time and space fractional solution of Burger's equations [14-15]. Asaithambi, Hon, Mao, Asaithambi and Mena studied about Burger's equations with using different methods [9, 10 and 11].

\section{Numerical method}

\section{Fractional Calculus:}

We can define the fractional calculus as the expand of differential and integral terms with non-integer orders. Caputo and Riemann-Liouville fractional derivatives are used in the approximation of the solving of partial differential equations $[2,19]$.

\section{Gamma Function:}

The gamma function is the expand of factorial to real numbers. The following expression is general form of gamma function;

$$
\Gamma(z)=\int_{0}^{\infty} e^{-u} u^{z-1} d u, \quad z \in R
$$

\section{Riemann-Liouville fractional derivative:}

We know the following expression as Cauchy integral

$$
\int_{a}^{x} d t_{1} \int_{a}^{t_{1}} d t_{2} \ldots \int_{a}^{t_{n-1}} f\left(t_{n}\right) d t_{n}=\frac{1}{\Gamma(n)} \int_{a}^{x}(x-t)^{n-1} f(t) d t
$$

If we change the $\mathrm{n}$ term with $\mathrm{q}$, the q can be a real number; we can achieve the RiemannLiouville fractional integral;

$$
\frac{d^{q} f}{d(x-a)^{q}}=\frac{1}{\Gamma(-q)} \int_{a}^{x}(x-t)^{-q-1} f(t) d t \quad ; q<0
$$

With some changings in the Equation (5) we can achieve the Riemann-Liouville fractional derivative: 


$$
\frac{d^{q} f}{d(x-a)^{q}}=\frac{d^{n}}{d x^{n}}\left[\frac{1}{\Gamma(n-q)} \int_{a}^{x}(x-t)^{-(q-n)-1} f(t) d t\right]
$$

In this expression $n>q$ and $q \geq 0$.

\section{Caputo Fractional derivative:}

The other approach of fractional calculus is caputo's approach. If we want to use physical conditions effectively we can use Caputo fractional derivative. The physical conditions are same in integer orders between Caputo and normal derivative. The following equation is a general form of Caputo fractional derivative:

$$
{ }_{a}^{C} D_{t}^{\alpha}=\frac{1}{\Gamma(\alpha-n)} \int_{a}^{t} \frac{f^{(n)}(\tau) d \tau}{(t-\tau)^{\alpha+1-n}}, \quad(n-1<\alpha<n)
$$

\section{Finite Difference Method:}

In our approximation we use the following forms of Caputo and Riemann-Liouville fractional derivatives:

Caputo fractional derivative:

$$
\frac{\partial^{\alpha} u(x, t)}{\partial t^{\alpha}}=\frac{1}{\Gamma(1-\alpha)} \int_{0}^{t}(t-\eta)^{-\alpha} \frac{\partial u(x, \eta)}{\partial \eta} d \eta
$$

Riemann-Liouville derivative:

$$
\frac{\partial^{\beta} u(x, t)}{\partial t^{\beta}}=\frac{1}{\Gamma(2-\beta)} \frac{\partial^{2}}{\partial x^{2}} \int_{0}^{t} \frac{u(\xi, t)}{(x-\xi)^{\beta-1}} d \xi
$$

To find the finite difference scheme of fractional burgers equations, we choose grid size to $\Delta x$ for the space of this problem and we can find, then we can find integration time as $\tau=\frac{t}{n}$. $0<t_{k}<T$ for this problem, $(k=0,1, \ldots ., n)$ and $x_{i}=i h$ for this $\operatorname{problem}(i=0,1, \ldots ., m)$. In the scheme we write $U_{i}^{k}$ for $U\left(x_{i}, t_{k}\right)$. For writing the scheme we change time derivative term to time fractional derivative term;

$$
\begin{aligned}
\frac{\partial^{\alpha} u_{i}^{k+1}}{\partial t^{\alpha}} & =\frac{1}{\Gamma(1-\alpha)} \sum_{j=0}^{k} \frac{u_{i}^{j+1}-u_{i}^{j}}{\tau} \int_{j \tau}^{(j+1) \tau} \frac{d \gamma}{\left(t_{k+1}-\gamma\right)^{\alpha}}+o(\tau) \\
& =\frac{1}{\Gamma(2-\alpha)} \sum_{j=0}^{k} \frac{u_{i}^{k+1-j}-u_{i}^{k-j}}{\tau}\left[(j+1)^{1-\alpha}-j^{1-\alpha}\right]+O(\tau) .
\end{aligned}
$$

Then with using Riemann-Liouville fractional derivative, we can find the space fractional term of these problem as:

$$
\frac{\partial^{\beta} u_{i}^{k+1}}{\partial x^{\beta}}=\frac{1}{h^{\beta}} \sum_{j=0}^{i+1} g_{j} u_{i-(j-1) h}^{k+1}+O(\tau+h)
$$


In (10) $\mathrm{g}$ is a special function of $\beta$ and $j$, writing in (12). Finally with applying (9) and (10) to fractional Burger's equation [7-8], we can write the following finite difference scheme;

$$
\frac{\Delta t}{\Gamma(2-\alpha)} \sum_{j=0}^{k} \frac{u_{i}^{k+1-j}-u_{i}^{k-j}}{\Delta x}\left[(j+1)^{1-\alpha}-j^{1-\alpha}\right]=-u_{i}^{k} \frac{u_{i}^{k}-u_{i-1}^{k}}{\Delta x}+\frac{v}{(\Delta x)^{\beta}} \sum_{j=0}^{i+1} g_{i} u_{i-j+1}^{k+1} \text {. }
$$

Take some terms as special terms for convenience[18].

$$
\begin{aligned}
& \sigma_{j}=(j+1)^{1-\alpha}-j^{1-\alpha} \\
& p_{i}=\frac{\Gamma(2-\alpha)(\Delta t)^{\alpha}}{\Delta x} \\
& r_{i}=\frac{v}{(\Delta x)^{\beta}}(\Delta t)^{\alpha} \Gamma(2-\alpha) \\
& g_{j}=(-1)^{j} \frac{\beta \cdot(\beta-1) \cdot(\beta-2) \ldots \ldots(\beta-j+1)}{j !}, \quad g_{0}=1, \quad g_{1}=-\beta .
\end{aligned}
$$

If we write this changing, we can achieve the following scheme;

$$
\sum_{j=0}^{k} \sigma_{j}\left(u_{i}^{k+1-j}-u_{i}^{k-j}\right)=-p_{i}\left(u_{i}^{k}\left(u_{i}^{k}-u_{i-1}^{k}\right)\right)+r_{i} \sum_{j=0}^{i+1} g_{j} u_{i-j+1}^{k+1} \text {. }
$$

For $k=0$ we can get this expression;

$$
\begin{gathered}
u_{i}^{1}-u_{i}^{0}+p_{i}\left(u_{i}^{0}\left(u_{i}^{0}-u_{i-1}^{0}\right)\right)=r_{i} \sum_{j=0}^{i+1} g_{i} u_{i-j+1}^{1} \cdot \\
u_{i}^{1}-u_{i}^{0}+p_{i}\left(u_{i}^{0}\left(u_{i}^{0}-u_{i-1}^{0}\right)\right)=r_{i} u_{i+1}^{1}+r_{i} u_{i}^{1} g_{1}+r_{i} g_{2} u_{i-1}^{1}+r_{i} \sum_{j=3}^{i+1} g_{i} u_{i-j+1}^{1} . \\
-r_{i} u_{i+1}^{1}+\left(1-r_{i} g_{1}\right) u_{i}^{1}-r_{i} g_{2} u_{i-1}^{1}-r_{i} \sum_{j=3}^{i+1} g_{i} u_{i-j+1}^{1} u_{i}^{1}=-p_{i}\left(u_{i}^{0}\left(u_{i}^{0}-u_{i-1}^{0}\right)\right)+u_{i}^{0} .
\end{gathered}
$$

Then for $k>0$, we can get this expression;

$$
\begin{aligned}
\sigma_{0}\left(u_{i}^{k+1}-u_{i}^{k}\right)+\sum_{j=1}^{k} \sigma_{j} u_{i}^{k+j-1}-u_{i}^{k-j}+p_{i}\left(u_{i}^{k}\left(u_{i}^{k}-u_{i-1}^{k}\right)=r_{i} u_{i+1}^{k+1}\right. & +r_{i} g_{1} u_{i}^{k+1}+r_{i} g_{2} u_{i-1}^{k+1} \\
& +r_{i} \sum_{j=3}^{i+1} g_{j} u_{i-j+1}^{k+1} .
\end{aligned}
$$

If we take $p_{i}\left(u_{i}^{k}\left(u_{i}^{k}-u_{i-1}^{k}\right)\right.$ and do some regulations, 


$$
\begin{aligned}
-r_{i} u_{i+1}^{k+1}+\left(1-r_{i} g_{1}\right) u_{i}^{k+1}-r_{i} g_{2} u_{i-1}^{k+1}-r_{i} \sum_{j=3}^{i+1} g_{j} u_{i-j+1}^{k+1}=u_{i}^{k}-\sum_{j=1}^{k} \sigma_{j}\left(u_{i}^{k+j-1}-u_{i}^{k-j}\right)-P & \\
= & u_{i}^{k}-\sum_{j=1}^{k} \sigma_{j} u_{i}^{k+j-1}+\sum_{j=1}^{k} \sigma_{j} u_{i}^{k-j}-P \\
& =u_{i}^{k}-\sum_{j=0}^{k-1} \sigma_{j+1} u_{i}^{k+j}+\sum_{j=1}^{k} \sigma_{j} u_{i}^{k-j}-P . \\
-r_{i} u_{i+1}^{k+1}+\left(1-r_{i} g_{1}\right) u_{i}^{k+1}-r_{i} g_{2} u_{i-1}^{k+1}-r_{i} \sum_{j=3}^{i+1} g_{j} u_{i-j+1}^{k+1}=u_{i}^{k}- & \sigma_{1} u_{i}^{k}+\sigma_{k} u_{i}^{0}+\sum_{j=1}^{k-1}\left(-\sigma_{j+1}+\sigma_{j}\right) u_{i}^{k-j}-P \\
= & \sigma_{k} u_{i}^{0}+\left(2-2^{1-\alpha}\right) u_{i}^{k} \\
& +\sum_{j=1}^{k-1} u_{i}^{k-j}\left(2(j+1)^{1-\alpha}-(j+2)^{1-\alpha}-j^{1-\alpha}\right)-P .
\end{aligned}
$$

Finally for $k>0$ our difference scheme is;

$$
\begin{aligned}
-r_{i} u_{i+1}^{k+1}+\left(1-r_{i} g_{1}\right) u_{i}^{k+1}-r_{i} g_{2} u_{i-1}^{k+1}-r_{i} \sum_{j=3}^{i+1} g_{j} u_{i-j+1}^{k+1}= & \sigma_{k} u_{i}^{0}+\left(2-2^{1-\alpha}\right) u_{i}^{k} \\
& +\sum_{j=1}^{k-1} u_{i}^{k-j}\left(2(j+1)^{1-\alpha}-(j+2)^{1-\alpha}-j^{1-\alpha}\right) \\
& -p_{i}\left(u_{i}^{k}\left(u_{i}^{k}-u_{i-1}^{k}\right) .\right.
\end{aligned}
$$

If we want to write this difference scheme as a algebraic equation system;

$$
\left\{\begin{array}{l}
A U^{1}=-p_{i} u_{i}^{0}\left(u_{i}^{0}-u_{i-1}^{0}\right)+u_{i}^{0} \\
A U^{k+1}=d_{1} U^{k}+d_{2} U^{k-1}+\ldots .+d_{k} U^{1}+\sigma_{k} U^{0}-p_{i}\left(u_{i}^{k}\left(u_{i}^{k}-u_{i-1}^{k}\right), \quad k>0\right. \\
U^{0}=f
\end{array}\right.
$$

In this system;

$$
\begin{aligned}
d_{j} & =2 j^{1-\alpha}-(j+1)^{1-\alpha}-(j-1)^{1-\alpha}, \quad j=1,2, \ldots . ., k \\
\sigma_{j} & =(j+1)^{1-\alpha}-j^{1-\alpha} \\
p_{i} & =\frac{\Gamma(2-\alpha)(\Delta t)^{\alpha}}{\Delta x}
\end{aligned}
$$

$\mathrm{A}$ is a matrix which has the coefficient of unknown terms for our problem;

$$
A_{i j}= \begin{cases}-r_{i} g_{i-j+1}, & j<i-1 \\ -r_{i} g_{2}, & j=i-1 \\ 1-r_{i} g_{1}, & j=i \\ -r_{i}, & j=i+1 \\ 0, & j>i+1\end{cases}
$$

In this matrix; 


$$
\begin{aligned}
& r_{i}=\frac{v}{(\Delta x)^{\beta}}(\Delta t)^{\alpha} \Gamma(2-\alpha) \\
& g_{j}=(-1)^{j} \frac{\beta \cdot(\beta-1) \cdot(\beta-2) \ldots \ldots(\beta-j+1)}{j !}, \quad g_{0}=1, \quad g_{1}=-\beta
\end{aligned}
$$

An example for Aij matrix for $\mathrm{i}$ and $\mathrm{j}$ from 1 to 10 ;

$$
A_{i j}=\left(\begin{array}{cccccccccc}
1-r_{i} g_{1} & -r_{i} & 0 & 0 & 0 & 0 & 0 & 0 & 0 & 0 \\
-r_{i} g_{2} & 1-r_{i} g_{1} & -r_{i} & 0 & 0 & 0 & 0 & 0 & 0 & 0 \\
-r_{i} g_{3} & -r_{i} g_{2} & 1-r_{i} g_{1} & -r_{i} & 0 & 0 & 0 & 0 & 0 & 0 \\
-r_{i} g_{4} & -r_{i} g_{3} & -r_{i} g_{2} & 1-r_{i} g_{1} & -r_{i} & 0 & 0 & 0 & 0 & 0 \\
-r_{i} g_{5} & -r_{i} g_{4} & -r_{i} g_{3} & -r_{i} g_{2} & 1-r_{i} g_{1} & -r_{i} & 0 & 0 & 0 & 0 \\
-r_{i} g_{6} & -r_{i} g_{5} & -r_{i} g_{4} & -r_{i} g_{3} & -r_{i} g_{2} & 1-r_{i} g_{1} & -r_{i} & 0 & 0 & 0 \\
-r_{i} g_{7} & -r_{i} g_{6} & -r_{i} g_{5} & -r_{i} g_{4} & -r_{i} g_{3} & -r_{i} g_{2} & 1-r_{i} g_{1} & -r_{i} & 0 & 0 \\
-r_{i} g_{8} & -r_{i} g_{7} & -r_{i} g_{6} & -r_{i} g_{5} & -r_{i} g_{4} & -r_{i} g_{3} & -r_{i} g_{2} & 1-r_{i} g_{1} & -r_{i} & 0 \\
-r_{i} g_{9} & -r_{i} g_{8} & -r_{i} g_{7} & -r_{i} g_{6} & -r_{i} g_{5} & -r_{i} g_{4} & -r_{i} g_{3} & -r_{i} g_{2} & 1-r_{i} g_{1} & -r_{i} \\
-r_{i} g_{10} & -r_{i} g_{9} & -r_{i} g_{8} & -r_{i} g_{7} & -r_{i} g_{6} & -r_{i} g_{5} & -r_{i} g_{4} & -r_{i} g_{3} & -r_{i} g_{2} & 1-r_{i} g_{1}
\end{array}\right)
$$

\section{Stability and uniqueness}

Theorem 3.1 The implicit system defined by the linear difference equations (19) and (20) has a unique solution and is unconditionally stable for all $0<\alpha<1,1<\beta<2$.

Proof. By applying the Gerschgorin theorem we decided that each eigenvalue of matrix A had a magnitude greater than 1 .

Note this $g_{0}=1, g_{1}=-\beta, g_{j}=(-1)^{j} \frac{\beta(\beta-1) \ldots(\beta-j+1)}{j !}, j=1,2,3, \ldots$, then for $1<\beta \leq 2$, and $j \geq 2$, we have $g_{j} \geq 0$. Also, with well-known results that for any $\gamma>0$,

$$
(1+z)^{\gamma}=\sum_{m=0}^{\infty}\left(\begin{array}{l}
\gamma \\
m
\end{array}\right) z^{m},|z| \leq 1
$$

Substituting $z=-1$ into (26) yields $\sum_{j=0}^{\infty} g_{j}=0$, and then $-g_{1}>\sum_{j=0, j \neq 1}^{\infty} g_{j}$, i.e. $\sum_{j=0}^{\infty} g_{j}<0$ for any $I=1,2,3, \ldots . m$. According to the Gerschgorin theorem, the eigenvalues of the matrix $A$ are in the disks centered at $A_{i, i}=1-r_{i} g_{1}=1+r_{i} \beta$ with radius

$$
R_{i}=\sum_{j=1, j \neq 1}^{m-1}\left|A_{i, j}\right|=\sum_{j=1, j \neq 1}^{i+1}\left|A_{i, j}\right|=\sum_{j=1}^{i-2}\left|-r_{i} g_{i-j+1}\right|+\left|-r_{i} g_{2}\right|+\left|-r_{i}\right|=r_{i} \sum_{j=0, j \neq 1}^{i-2} g_{j}<-r_{i} g_{1} \leq r_{i} \beta .
$$


Hence, each eigenvalue $\lambda$ of the matrix $A$ has a real part which is greater than one, and therefore has a magnitude greater than one. Therefore, the spectral radius of $A^{-1}$ is less than one. This proves that the scheme has a unique solution.

To prove unconditional stability of (19) and (20) let $u_{i}^{k}, \tilde{u}_{i}^{k}(i=1,2,3, \ldots, m-1, k=1,2, \ldots n-1)$ be the solution of (19) and (20) with initial value $u_{i}^{0}, \tilde{u}_{i}^{0}$ respectively, the computation of $q_{i}^{k}(i=1,2, \ldots, m-1, k=1,2, \ldots, n-1)$ is exact. Then error $\varepsilon_{i}^{k}=\tilde{u}_{i}^{k}-u_{i}^{k}$ satisfies if $k=0$,

$$
-r_{i} \varepsilon_{i+1}^{1}+\left(1-r_{i} g_{1}\right) \varepsilon_{i}^{1}-\left(r_{i} g_{2}\right) \varepsilon_{i-1}^{1}-r_{i} \sum_{j=3}^{i+1} g_{i} \varepsilon_{i-j+1}^{1}=\varepsilon_{i}^{0}
$$

if $k>0$,

$$
-r_{i} \varepsilon_{i+1}^{k+1}+\left(1-r_{i} g_{1}\right) \varepsilon_{i}^{k+1}-\left(r_{i} g_{2}\right) \varepsilon_{i-1}^{k+1}-r_{i} \sum_{j=3}^{i+1} g_{i} \varepsilon_{i-j+1}^{k+1}=d_{1} \varepsilon_{i}^{k}+\sum_{j=1}^{k-1} d_{j+1} \varepsilon_{i}^{k-j}+\sigma_{k} \varepsilon_{i}^{0}
$$

Equivalent to the following matrix form:

$$
A E^{1}=E^{0}, A E^{k+1}=d_{1} E^{k}+d_{2} E^{k-1}+\ldots+d_{k} E^{1}+\sigma_{k} E^{0}, k>0
$$

where $E^{k}=\left(\varepsilon_{1}^{k}, \varepsilon_{2}^{k}, \ldots, \varepsilon_{m-1}^{k}\right)^{T}$. Let us use mathematical induction to prove $\left\|E^{k}\right\|_{\infty} \unlhd\left\|E^{0}\right\|_{\infty}, k=1,2, \ldots$ In fact, if $\mathrm{k}=1$, suppose $\left|\varepsilon_{l}^{1}\right|=\max _{1 \leq i \leq m-1}\left|\varepsilon_{i}^{1}\right|$, note that $r_{i}, p_{i}>0$ and for any integer number $N, \sum_{j=0}^{\infty} g_{j}<0$, we have

$$
\begin{aligned}
\left\|E^{1}\right\|_{\infty} & =\left|\varepsilon_{l}^{1}\right| \leq\left|\varepsilon_{l}^{1}\right|+p_{l}\left(\left|\varepsilon_{l}^{1}\right|-\left|\varepsilon_{l-1}^{1}\right|\right)-r_{l}\left(\sum_{j=0}^{l+1} g_{j}\right)\left|\varepsilon_{l}^{1}\right| \\
& \leq-r_{l}\left|\varepsilon_{l}^{1}\right|+\left(1-r_{l} g_{1}\right)\left|\varepsilon_{l}^{1}\right|-\left(r_{l} g_{2}\right)\left|\varepsilon_{l-1}^{1}\right|-r_{l}\left(\sum_{j=3}^{l+1} g_{j}\right)\left|\varepsilon_{l-j+1}^{1}\right| \\
& \leq-r_{l} \varepsilon_{l}^{1}+\left(1-r_{l} g_{1}\right) \varepsilon_{l}^{1}-\left(r_{l} g_{2}\right) \varepsilon_{l-1}^{1}-r_{l}\left(\sum_{j=3}^{l+1} g_{j}\right) \varepsilon_{l-j+1}^{1}|=| \varepsilon_{l}^{0}|\leq| \mid E^{0} \| .
\end{aligned}
$$

Therefore $\left\|E^{1}\right\|_{\infty} \unlhd\left\|E^{0}\right\|_{\infty}$. Suppose if $k \leq s,\left\|E^{s}\right\|_{\infty} \unlhd\left\|E^{0}\right\|_{\infty}$ hold, then when $k=s+1$, let $\left|\varepsilon_{l}^{s+1}\right|=\max _{1 \leq i \leq m-1}\left|\varepsilon_{i}^{s+1}\right|$, notice that $\sum_{j=0}^{i} g_{j}<0, i=1,2, \ldots, m$ similar to previous estimate, we have 


$$
\begin{aligned}
\left\|E^{s+1}\right\|_{\infty} & =\left|\varepsilon_{l}^{s+1}\right| \leq-r_{l}\left|\varepsilon_{l+1}^{s+1}\right|+\left(1-r_{l} g_{1}\right)\left|\varepsilon_{l}^{s+1}\right|-\left(r_{l} g_{2}\right)\left|\varepsilon_{l-1}^{s+1}\right|-r_{l} \sum_{j=3}^{l+1} g_{j}\left|\varepsilon_{l-j+1}^{s+1}\right| \\
& \leq-r_{l} \varepsilon_{l+1}^{s+1}+\left(1-r_{l} g_{1}\right) \varepsilon_{l}^{s+1}-\left(r_{l} g_{2}\right) \varepsilon_{l-1}^{s+1}-r_{l} \sum_{j=3}^{l+1} g_{j} \varepsilon_{l-j+1}^{s+1} \mid \leq\left\|A E^{s+1}\right\|_{\infty} \\
& \leq d_{1}\left|\varepsilon_{l}^{s}\right|+\sum_{j=1}^{s-1} d_{j+1}\left|\varepsilon_{l}^{s-j}\right|+\left|\varepsilon_{l}^{0}\right| \cdot\left[(s+1)^{1-\alpha}-s^{1-\alpha}\right] \\
& \leq d_{1}\left\|E^{s-j}\right\|_{\infty}+\sum_{j=1}^{s-1} d_{j+1}\left\|E^{s-j}\right\|_{\infty}+\left[(s+1)^{1-\alpha}-s^{1-\alpha}\right] \cdot\left\|E^{0}\right\|_{\infty} \\
& \leq\left(d_{1}+\sum_{j=1}^{s-1} d_{j+1}+\left[(s+1)^{1-\alpha}-s^{1-\alpha}\right]\right) \cdot\left\|E^{0}\right\|_{\infty}=\left\|E^{0}\right\|_{\infty}
\end{aligned}
$$

Hence, $\left\|E^{s+1}\right\|_{\infty} \triangleleft\left\|E^{0}\right\|_{\infty}$ so the implicit scheme defined by the linear difference equations (19) and (20) is unconditionally stable and Theorem 3.1 completes the proof.

Denote $e_{i}^{k}=u\left(x_{i}, t^{k}\right)-u_{i}^{k}$ and $e^{k}=\left(e_{1}^{k}, e_{2}^{k}, \ldots, e_{m-1}^{k}\right)^{T}$, we have Theorem 3.2.

Theorem 2. Suppose that $u\left(x_{i}, t_{k}\right)$ is the exact solution of (1) at grid point $\left(x_{i}, t_{k}\right), u_{i}^{k}$ is the difference solution of (19), (20), then there exists positive constant $M$, such that

$$
\left\|e^{k}\right\|_{\infty} \leq \sigma_{k-1}^{-1} M\left(\tau^{1+\alpha}-\tau^{\alpha} h\right), k=1,2, \ldots, n
$$

where $\left\|e^{k}\right\|_{\infty}=\max _{1 \leq i \leq m-1}\left|e_{i}^{k}\right|, M$ is a constant independent of $h$ and $\tau$.

Proof. Since $u_{i}^{k}=u\left(x_{i}, t^{k}\right)-e_{i}^{k}$, notice that $e^{0}=0$, we have from (19) and (20), if $k=0$,

$$
-r_{i} e_{i+1}^{1}+\left(1-r_{i} g_{1}\right) e_{i}^{1}-\left(r_{i} g_{2}\right) e_{i-1}^{1}-r_{i} \sum_{j=3}^{i+1} g_{i} e_{i-j+1}^{1}=R_{i}^{1}
$$

if $k>0$,

$$
-r_{i} e_{i+1}^{k+1}+\left(1-r_{i} g_{1}\right) e_{i}^{k+1}-\left(r_{i} g_{2}\right) e_{i-1}^{k+1}-r_{i} \sum_{j=3}^{i+1} g_{i} e_{i-j+1}^{k+1}=d_{1} e_{i}^{k}+\sum_{j=1}^{k-1} d_{j+1} e_{i}^{k-j}+R_{i}^{k+1},
$$

where $\left\|R_{i}^{k+1}\right\|_{\infty} \leq M\left(\tau^{1+\alpha}-\tau^{\alpha} h\right), i=1,2, \ldots, m-1, k=1,2, \ldots, n-1, \quad M$ is positive constant independent of $h$ and $\tau$. Let's use mathematical induction to prove the theorem. If $k=1$, suppose $\left\|e_{l}^{1}\right\|_{\infty}=\max _{1 \leq i \leq m-1}\left|e_{l}^{1}\right|$, we have 


$$
\begin{aligned}
\left\|e^{1}\right\|_{\infty} & =\left|e_{l}^{1}\right| \leq-r_{l}\left|e_{l+1}^{1}\right|+\left(1-r_{l} g_{1}\right)\left|e_{l}^{1}\right|-\left(r_{l} g_{2}\right)\left|e_{l-1}^{1}\right|-r_{l} \sum_{j=3}^{l+1} g_{j}\left|e_{l-j+1}^{1}\right| \\
& \leq-r_{l} e_{l+1}^{1}+\left(1-r_{l} g_{1}\right) e_{l}^{1}-\left(r_{l} g_{2}\right) e_{l-1}^{1}-r_{l} \sum_{j=3}^{l+1} g_{j} e_{l-j+1}^{1}|=| R_{l}^{1} \mid \leq M\left(\tau^{1+\alpha}+\tau^{\alpha} h\right)=\sigma_{0}^{-1} M\left(\tau^{1+\alpha}+\tau^{\alpha} h\right) .
\end{aligned}
$$

Suppose that if $k \leq s,\left\|e^{s}\right\|_{\infty} \leq \sigma_{s-1}^{-1} M\left(\tau^{1+\alpha}+\tau^{\alpha} h\right)$ hold, then when $k=s+1$, let $\left|e_{l}^{s+1}\right|=\max _{1 \leq i \leq m-1}\left|e_{i}^{s+1}\right|$, notice that $\sigma_{j}^{-1}<\sigma_{k}^{-1}, j=0,1,2, \ldots, k$ and $\sum_{j=0}^{i} g_{j}<0, i=1,2, \ldots, m$ Therefore

$$
\begin{aligned}
\left\|e^{s+1}\right\|_{\infty} & =\left|e_{l}^{s+1}\right| \leq d_{1}\left\|e^{s}\right\|_{\infty}+\sum_{j=1}^{s-1} d_{j+1}\left\|e^{s-j}\right\|_{\infty}+M\left(\tau^{1+\alpha}+\tau^{\alpha} h\right)=\sum_{j=0}^{s-1} d_{j+1}\left\|e^{s-j}\right\|_{\infty}+M\left(\tau^{1+\alpha}+\tau^{\alpha} h\right) \\
& \leq\left(d_{1} \sigma_{s-1}^{-1}+d_{2} \sigma_{s-2}^{-1}+\ldots+d_{s} \sigma_{0}^{-1}+1\right) M\left(\tau^{1+\alpha}+\tau^{\alpha} h\right) \\
& \leq \sigma_{s}^{-1}\left(\sum_{i=0}^{s-1} d_{i+1}+\sigma_{s}\right) M\left(\tau^{1+\alpha}+\tau^{\alpha} h\right)=\sigma_{s}^{-1} M\left(\tau^{1+\alpha}+\tau^{\alpha} h\right) .
\end{aligned}
$$

Therefore Theorem 3.2 is proved.

\section{Numerical examples}

\section{Example 4.1}

$$
\begin{aligned}
& \frac{\partial^{\alpha} u}{\partial t^{\alpha}}+u \frac{\partial u}{\partial x}=v \frac{\partial^{\beta} u}{\partial x^{\beta}} \quad 0 \leq x \leq 1,0<t \leq 0,1, \\
& 0<\alpha<1,1<\beta<2
\end{aligned}
$$$$
\text { boundary condutions: }
$$$$
u(0, t)=0, \quad u(1, t)=0
$$

initial condution:

$$
u(x, 0)=\frac{2 v \pi \sin (\pi x)}{a+\cos (\pi x)}, \quad a>1
$$

We can solve numerically this problem in following conditions,

$$
v=0.001, \quad a=1.1, \quad \alpha=0.95, \quad \beta=1.95
$$

Then the analytical solution of Example 4.1 is:

$$
u(x, t)=\frac{2 v \pi \exp \left(-\pi^{2} v t\right) \sin (\pi x)}{a+\exp \left(-\pi^{2} v t\right) \cos (\pi x)}
$$




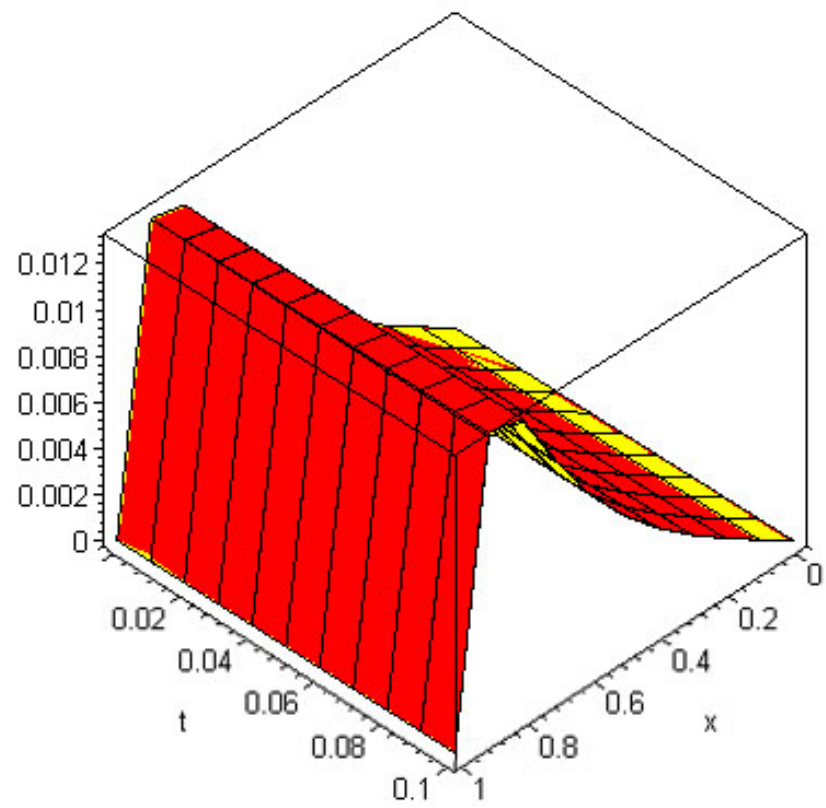

Analytical Solution

Numerical Solution

Finite difference solution for Example 4.1 for $\Delta t=0.01, \Delta x=0.1, \alpha=0.95, \beta=1.95$

\begin{tabular}{llll}
\hline $\mathrm{X}$ & Numerical Solution & Analytical Solution & Error \\
\hline 0 & 0.0 & 0.0 & 0.0 \\
0.1 & $0.946184518 \mathrm{e}-3$ & $0.94613841 \mathrm{e}-3$ & $0.461089 \mathrm{e}-7$ \\
0.2 & $0.1924465410 \mathrm{e}-2$ & $0.1933489085 \mathrm{e}-2$ & $0.9023675 \mathrm{e}-5$ \\
0.3 & $0.2991456650 \mathrm{e}-2$ & $0.3009822748 \mathrm{e}-2$ & $0.18366098 \mathrm{e}-4$ \\
0.4 & $0.4209350415 \mathrm{e}-2$ & $0.4237749682 \mathrm{e}-2$ & $0.28399267 \mathrm{e}-4$ \\
0.5 & $0.5666712399 \mathrm{e}-2$ & $0.5706351919 \mathrm{e}-2$ & $0.3963952 \mathrm{e}-4$ \\
0.6 & $0.7491462912 \mathrm{e}-2$ & $0.7544371558 \mathrm{e}-2$ & $0.52908646 \mathrm{e}-4$ \\
0.7 & $0.9832247233 \mathrm{e}-2$ & $0.99029697 \mathrm{e}-1$ & $0.70722543 \mathrm{e}-4$ \\
0.8 & $0.1253842757 \mathrm{e}-1$ & $0.1264482419 \mathrm{e}-1$ & $0.10639662 \mathrm{e}-3$ \\
0.9 & $0.1278084698 \mathrm{e}-1$ & $0.1294151322 \mathrm{e}-1$ & $0.16066624 \mathrm{e}-3$ \\
\hline 1 & 0.0 & 0.0 & 0.0 \\
\hline
\end{tabular}

\section{Example 4.2}

$$
\begin{aligned}
& \frac{\partial^{\alpha} u}{\partial t^{\alpha}}+u \frac{\partial u}{\partial x}=v \frac{\partial^{\beta} u}{\partial x^{\beta}} \quad 0 \leq x \leq 1,0<t \leq 0,01, \\
& 0<\alpha<1,1<\beta<2
\end{aligned}
$$

boundary condutions :

$u(0, t)=0, \quad u(1, t)=0$

initial condution:

$u(x, 0)=4 x(1-x)$

We can solve numerically this problem in following conditions, 


$$
v=0.001, \quad \alpha=0.95, \quad \beta=1.95
$$

Then the analytical solution of Example 4.2 is:

$$
u(x, t)=\frac{2 v \pi \sum_{k=1}^{\infty} k A_{k} \exp \left(-k^{2} \pi^{2} v t\right) \sin (k \pi x)}{A_{0}+2 v \pi \sum_{k=1}^{\infty} k A_{k} \exp \left(-k^{2} \pi^{2} v t\right) c \cos (k \pi x)},\left(\begin{array}{c}
A_{0}=\int_{0}^{1} \exp \left\{-x^{2}(3 v)^{-1}(3-2 x)\right\} d x \\
A_{k}=\int_{0}^{1} \exp \left\{-x^{2}(3 v)^{-1}(3-2 x)\right\} \cos (k \pi x) d x, k \geq 1
\end{array}\right)
$$

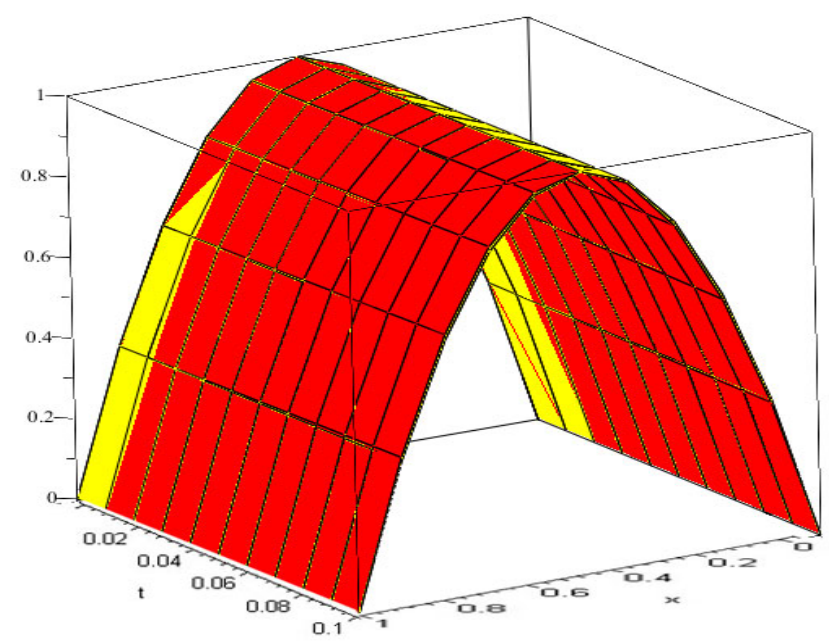

Analytical Solution

Numerical Solution

Finite difference solution for Example 4.2 for $\Delta t=0.001, \Delta x=0.1, \alpha=0.95, \beta=1.95$

\begin{tabular}{llll}
\hline $\mathbf{X}$ & Numerical Solution & Analytical Solution & Error \\
\hline $\mathbf{0}$ & 0.0 & 0.0 & 0.0 \\
$\mathbf{0 . 1}$ & 0.3473711502 & 0.3486899313 & $0.13187811 \mathrm{e}-2$ \\
$\mathbf{0 . 2}$ & 0.6220256101 & 0.6247583412 & $0.27327311 \mathrm{e}-2$ \\
$\mathbf{0 . 3}$ & 0.8226195405 & 0.8264408177 & $0.38212772 \mathrm{e}-2$ \\
$\mathbf{0 . 4}$ & 0.9474252601 & 0.9519845695 & $0.45593094 \mathrm{e}-2$ \\
$\mathbf{0 . 5}$ & 0.9946485647 & 0.9995746590 & $0.49260943 \mathrm{e}-2$ \\
$\mathbf{0 . 6}$ & 0.9624307453 & 0.9673304143 & $0.48996690 \mathrm{e}-2$ \\
$\mathbf{0 . 7}$ & 0.8488455983 & 0.8533022617 & $0.44566634 \mathrm{e}-2$ \\
$\mathbf{0 . 8}$ & 0.6518961906 & 0.6554683894 & $0.35721988 \mathrm{e}-2$ \\
$\mathbf{0 . 9}$ & 0.3695116587 & 0.3717305024 & $0.22188437 \mathrm{e}-2$ \\
$\mathbf{1}$ & 0.0 & 0.0 & 0.0 \\
\hline
\end{tabular}

\section{Acknowledgments}

Authors was partially supported by the Office of Scientific Research Projects of Yildiz Technical University (Project no: 2013-01-03-YL01). 


\section{References}

[1] Zhang, Y., "A finite difference method for fractional partial differential equation", Applied Mathematics and Computation 215 (2009) : 524-529.

[2] Podlubny, I., "Fractional differential equation", V198 of Mathematics in Science and Engineering, Academic Press, San Diego, (1999).

[3] Zhang, P.G., Wang, J.P., "A predictor-corrector compact finite difference scheme for Burgers' equation”, Applied Mathematics and Computation 219 (2012) : 892-898.

[4] Varöglu, E., Liam Finn, W.D., "Space-time finite elements incorporating characteristics for the burgers' equation”, Int. J. Numer. Methods Eng. 16 (1980) : 171-184.

[5] Kutluay, S., Bahadir, A.R., Özdes, A., "Numerical solution of one-dimensional burgers equation: explicit and exact-explicit finite difference methods", J. Comput. Appl. Math 103 (1999) : 251-261.

[6] Pandey, K., Verma, L.,. Verma, A.K, "On a finite difference scheme for burgers' equation", Appl. Math. Comput 215 (2009) : 2206-2214.

[7] Liao, W., "An implicit fourth-order compact finite difference scheme for onedimensional burgers' equation”, Appl. Math. Comput 206 (2008) : 755-764.

[8] Saria, M., Gürarslanb, G., "A sixth-order compact finite difference scheme to the numerical solutions of burgers' equation”, Appl. Math. Comput 208 (2009) : 475-483.

[9] Hon, Y., Mao, X., “An efficient numerical scheme for burgers' equation”, Appl. Math. Comput 95 (1998) : 37-50.

[10] Asaithambi, A., "Numerical solution of the burgers' equation by automatic differentiation", Appl. Math. Comput 216 (2010) : 2700-2708.

[11] Mena, S.A., Salim, B.C., "Ettahlil-lül adedil muadeleti burger biistihdamil frukatil müntahiyeti”, Mecelle-tül rafdin liulumil muhasebeti verriyaziyatil mücellidi, (2007) : $1-4$.

[12] Burgers, J.M., "A mathematical model illustrating the theory of turbulence”, Adv. Appl. Mech 1 (1948) : 171-199.

[13] Kurulay, M., Bayram, M., "Some properties of the Mittag-Leffler functions and their relation with the Wright functions", Advances in Difference Equations (2012) : 181.

[14] Kurulay, M., "The approximate and exact solutions of the space- and time-fractional Burgers equations", International Journal of Research and Reviews in Applied Sciences, 3 (2010) : 257-263.

[15] Momani, S., "Non-perturbative analytical solutions of the space and time fractional Burgers equations", Chaos, Solutions and Fractals, 28 (4) (2006) : 930-937.

[16] Bashan, A., Karakoc, S.B.G., Geyikli, T., "Approximation of the KdVB equation by the quintic B-spline differential quadrature method", Kuwait journal of science, 42 (2) (2015) : 67-92.

[17] Y1lmaz, S., Ünlütürk Y., Mağden, A., "A study on the characterizations of non-null curves according to the Bishop frame of type-2 in Minkowski 3-space", SAÜ Fen Bil Der 20 (2) (2016) : 325-335.

[18] Meerschaert, M.M., Tadjeran, C., "Finite difference approximations for fractional advection-dispersion flow equations", J. Comput. Appl. Math. 172 (2003) : 65-77. 
[19] Meerschaert, M.M., Scheffler, H.P., Tadjeran, C., "Finite difference method for two dimensional fractional dispersion equation", J. Comput. Phys. 211 (2006) : 249-261. 